\title{
On the Frobenius vector of some simplicial affine semigroups
}

\author{
Ali Mahdavi $\quad$ Farhad Rahmati*
}

\begin{abstract}
We give a formula for a Frobenius vector of a Gorenstein simplicial affine semigroup $S$, and when the semigroup is Cohen-Macaulay we give an algorithm computing the set of minimal Frobenius vectors of $S$ for a special class of semigroups.
\end{abstract}

\section{Introduction and basic notions}

The set of nonnegative integers will be denoted by $\mathbb{N}$. An affine semigroup is a finitely generated submonoid of $\mathbb{N}^{r}$ for some positive integer $r$. Let $S=$ $\left\langle a_{1}, \ldots, a_{r+m}\right\rangle$ be an affine semigroup generated by $A=\left\{a_{1}, \ldots, a_{r+m}\right\} \subset \mathbb{N}^{r}$, that is to say, $S=\mathbb{N} a_{1}+\mathbb{N} a_{2}+\cdots+\mathbb{N} a_{r+m}$. In such a case, $A$ will be said to be a system of generators of $S$. Moreover, if no proper subset of $A$ generates $S$, the set $A$ is a minimal system of generators of $S$. Every affine semigroup has a unique minimal system of generators (see [9, Chapter 3]). Let $K$ be a field. The ring $K[S]$ is defined as the subalgebra of $K\left[y_{1}, \ldots, y_{r}\right]$ generated by $y^{a_{1}}, y^{a_{2}}, \ldots$ and $y^{a_{r+m}}$ with $y^{\alpha}:=y_{1}^{\alpha_{1}} y_{2}^{\alpha_{2}} \cdots y_{r}^{\alpha_{r}}$ where $\alpha=\left(\alpha_{1}, \ldots, \alpha_{r}\right) \in \mathbb{N}^{r}$. The semigroup $S$ is said to be Cohen-Macaulay (Gorenstein) if $K[S]$ is. Let $I_{S}$, called the semigroup ideal of $S$, be the kernel of $K$-algebra homomorphism from $K\left[x_{1}, \ldots, x_{r+m}\right]$ to $K[S]$ defined by $x_{i} \mapsto y^{a_{i}}$. For $u=\left(u_{1}, \ldots, u_{r+m}\right) \in \mathbb{N}^{r+m}$, we define the $S$-degree of the monomial $x^{u}$ by $\sum_{i=1}^{r+m} u_{i} a_{i}$ and denote by $\operatorname{deg}_{S}\left(x^{u}\right)$. The semigroup $S$ is said to be complete intersection if $I_{S}$ is a complete intersection ideal. It is well-known that

\footnotetext{
${ }^{*}$ Corresponding author.

Received by the editors in February 2016 - In revised form in June 2016.

Communicated by S. Caenepeel.

2010 Mathematics Subject Classification : 20M05, 20M25, 11 D07.

Key words and phrases : Frobenius vector, simplicial affine semigroup.
} 
$I_{S}$ is a binomial prime ideal ([5, Proposition 1.4]). When $r=1$ and $a_{1}, \ldots, a_{m+1}$ are relatively prime positive integers, the semigroup is called numerical semigroup. In this case $\mathbb{N} \backslash S$ is a finite set. For a numerical semigroup $S$ the largest integer $f^{*}(S)$ in $\mathbb{N} \backslash S$ is called the Frobenius number of $S$, and the problem of finding this number is called the Frobenius problem. The Frobenius number occurs in many branches of mathematics and is one of the most studied invariants in the theory of numerical semigroups. This problem has attracted substantial attention in the last 100+ years (see [4], [7], [8]). There is no general formula for the Frobenius number for $m$ greater than one. Sylvester in [14] proved that for $m=1, f^{*}(S)=$ $a_{1} a_{2}-a_{1}-a_{2}$.

The Frobenius problem is generalized to the higher dimensional cases (see [1], [2], [15], [16]). The vector Frobenius problem of Cohen-Macaulay and Gorenstein simplicial affine semigroup is studied in the next section. It is shown that every simplicial affine semigroup has at least one minimal Frobenius vector and an algorithm is presented for computing minimal Frobenius vectors of some CohenMacaulay simplicial affine semigroup.

\section{Frobenius vector}

Let $S$ be the affine semigroup generated by $A=\left\{a_{1}, \ldots, a_{r+m}\right\}$ in $\mathbb{N}^{r}$ and $\mathbf{G}(S)$ be the group generated by $S$ in $\mathbb{Z}^{r}$, that is, $\mathbf{G}(S)=\{a-b \mid a, b \in S\}$. We use $\mathbf{G}\left(a_{1}, \ldots, a_{n}\right)$ to denote the group generated by $\left\{a_{1}, \ldots, a_{n}\right\}$.

Definition 1. The affine semigroup $S$ is called simplicial if there exist $a_{i_{1}}, \ldots, a_{i_{r}} \in A$ such that

(1) $a_{i_{1}}, \ldots, a_{i_{r}}$ are linearly independent over $\mathbb{Q}$ and

(2) for every $a \in S$, there exists $0 \neq n \in \mathbb{N}$ such that $n a \in \mathbb{N} a_{i_{1}}+\cdots+\mathbb{N} a_{i_{r}}$.

If $r$ is lesser than three, every affine semigroup is simplicial. From now on, we will suppose that $S$ is a simplicial affine semigroup. Assume without loss of generality that $\left\{i_{1}, \ldots, i_{r}\right\}=\{1, \ldots, r\}$. The Apéry set of $a \neq 0$ in $S$ is defined as $\operatorname{Ap}(S, a)=\{x \in S \mid x-a \notin S\}$. Let $k_{i}$ be the smallest natural number such that $k_{i} a_{r+i} \in \sum_{i=1}^{r} \mathbb{N} a_{i}$, for $i=1, \ldots m$. By definition,

$$
\bigcap_{i=1}^{r} \operatorname{Ap}\left(S, a_{i}\right) \subseteq\left\{\sum_{i=1}^{m} t_{i} a_{r+i} \mid 0 \leq t_{i}<k_{i}\right\},
$$

so $\cap_{i=1}^{r} \operatorname{Ap}\left(S, a_{i}\right)$ is finite. The set $\cap_{i=1}^{r} \operatorname{Ap}\left(S, a_{i}\right)$ is also called the Apéry set of $S$ relative to $E:=\left\{a_{1}, \ldots, a_{r}\right\}$. Observe that $\operatorname{Ap}(S, E):=\{s \in S \mid s-e \notin S, \forall e \in$ $E\}=\cap_{i=1}^{r} \operatorname{Ap}\left(S, a_{i}\right)$. The set $\left\{x^{\alpha} \mid \operatorname{deg}_{S}\left(x^{\alpha}\right) \in \operatorname{Ap}(S, E)\right\}$ is a basis for $\frac{K\left[x_{1}, \ldots, x_{r}+m\right]}{\left\langle I_{S}, x_{1}, \ldots, x_{r}\right\rangle}$ as a $K$-vector space.

The following proposition gives a useful criterion for determining whether or not a simplicial affine semigroup is Cohen-Macaulay (see [11, Corollary 1.6]).

Proposition 1. If $S$ is a simplicial affine semigroup, the following statements are equivalent:

- $K[S]$ is Cohen-Macaulay;

- For all $\omega_{1}, \omega_{2} \in \operatorname{Ap}(S, E)$, if $\omega_{1} \neq \omega_{2}$, then $\omega_{1}-\omega_{2} \notin \mathbf{G}\left(a_{1}, \ldots, a_{r}\right)$. 
By definition, every element $a \in S$ can be written as $a=\sum_{i=1}^{r} \alpha_{i} a_{i}+\omega$, with $\omega \in \operatorname{Ap}(\mathrm{S}, \mathrm{E})$ and $\alpha_{i} \in \mathbb{N}, i=1, \ldots, r$. Let $a=\sum_{i=1}^{r+m} z_{i} a_{i} \in \mathbf{G}(S)$ and $z_{k}<0$ for some $k \in\{r+1, \ldots, r+m\}$. Since $S$ is simplicial, there exists $n_{k} \in \mathbb{N}$ such that $\left(n_{k}-z_{k}\right) a_{k} \in \mathbb{N} a_{1}+\cdots+\mathbb{N} a_{r}$. So $a=\sum_{i=1, i \neq k}^{r+m} z_{i} a_{i}+\left(z_{k}-n_{k}\right) a_{k}+n_{k} a_{k}$ and $\left(z_{k}-n_{k}\right) a_{k} \in \mathbf{G}\left(a_{1}, \ldots, a_{r}\right)$. Repeating this process, we see that $a$ can be written as $\sum_{i=1}^{r} z_{i}^{\prime} a_{i}+\sum_{i=r+1}^{r+m} n_{i} a_{i}, n_{i} \in \mathbb{N}$. Without loss of generality one may assume $\sum_{i=r+1}^{r+m} n_{i} a_{i} \in \operatorname{Ap}(\mathrm{S}, \mathrm{E})$. Hence every element $a \in \mathbf{G}(S)$ can be written as $a=\sum_{i=1}^{r} z_{i} a_{i}+\omega$ where $\omega \in \operatorname{Ap}(\mathrm{S}, \mathrm{E})$. The next proposition, which is Corollary 1.7 from [11], assert that when $S$ is Cohen-Macaulay, this expression is unique.

Proposition 2. If $S$ is a Cohen-Macaulay simplicial affine semigroup, then

(1) Every element in $\mathbf{G}(S)$ is equal to an unique expression of the form $z_{1} a_{1}+\ldots+$ $z_{r} a_{r}+\omega$ with $z_{i} \in \mathbb{Z}$ and $\omega \in \operatorname{Ap}(\mathrm{S}, \mathrm{E})$.

(2) The element $\sum_{i=1}^{r} z_{i} a_{i}+\omega$ with $z_{i} \in \mathbb{Z}$ and $\omega \in \operatorname{Ap}(\mathrm{S}, \mathrm{E})$ is in $S$ if and only if $z_{i} \geq 0$ for all $i$.

The cone spanned by $S$ and interior of cone $S$, are denoted by:

$$
\operatorname{cone}(S)=\left\{\sum_{i=1}^{r} r_{i} a_{i} \mid r_{i} \in \mathbb{Q}_{\geq 0}\right\}, \text { intcone }(S)=\left\{\sum_{i=1}^{r} r_{i} a_{i} \mid r_{i} \in \mathbb{Q}_{>0}\right\}
$$

respectively. From the definition it is easy to see that

$$
\mathbf{G}(S) \cap \operatorname{intcone}(S)=\left\{\sum_{i=1}^{r} z_{i} a_{i}+\omega_{j} \mid \gamma_{1}^{j}>-z_{1}, \ldots, \gamma_{r}^{j}>-z_{r}, j=1, \ldots, t\right\} .
$$

Definition 2. Let $S$ be an affine semigroup. The vector $f^{*} \in \mathbf{G}(S) \backslash S$ is called a Frobenius vector for $S$ if for all $x \in \mathbf{G}(S) \cap \operatorname{intcone}(S), f^{*}+x \in S$.
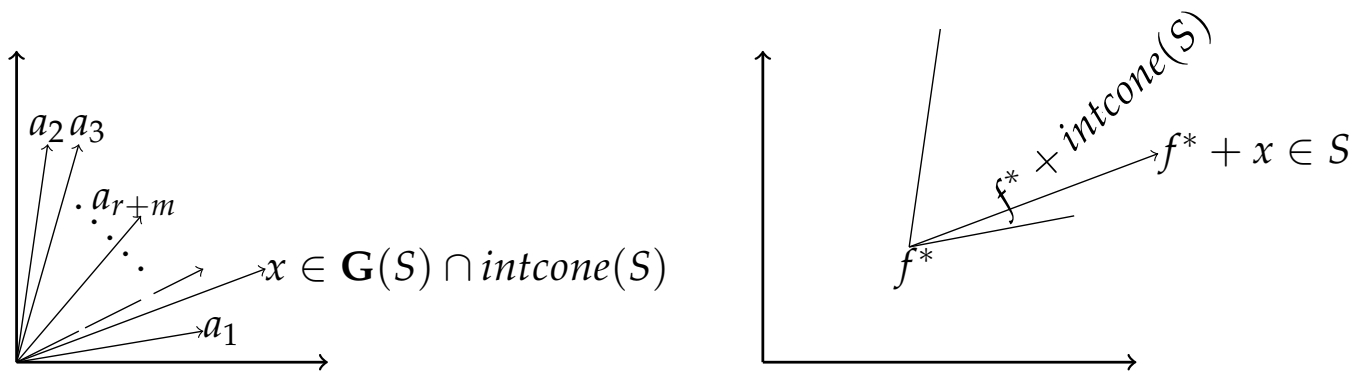

The set of Frobenius vectors of $S$ will be denoted by $\mathrm{F}(\mathrm{S})$. We define a cone ordering on $\mathrm{F}(\mathrm{S})$ by writing $f_{1}^{*} \leq f_{2}^{*}$ if $f_{2}^{*}+\operatorname{cone}(S) \subseteq f_{1}^{*}+\operatorname{cone}(S)$. We will denote by $\operatorname{MF}(S)$ the set of minimal Frobenius vectors of $S$ with respect to $\leq$.

Let $\operatorname{Ap}(S, E)=\left\{\omega_{1}=0, \omega_{2}, \ldots, \omega_{t}\right\}$. Since $S$ is simplicial, there exist nonnegative rational numbers $\gamma_{i}^{j}, i=1, \ldots, r, j=1, \ldots, t$, such that $\omega_{j}=\sum_{i=1}^{r} \gamma_{i}^{j} a_{i}$. Let $M$ and $M_{i}^{j}$ are $r \times r$ matrices, with column vectors $a_{1}, a_{2}, \ldots, a_{r}$ and $a_{1}, a_{2}, \ldots, \hat{a}_{i}, \ldots, a_{r}, \omega_{j}$, respectively, where $\hat{a}_{i}$ means that $a_{i}$ is omitted. It is not hard to see that

$$
\gamma_{i}^{j}=\left|\frac{\operatorname{det} M_{i}^{j}}{\operatorname{det} M}\right|
$$


Now, by Euclidean division, there exists a unique integer $\mu_{i}^{j} \geq-1$ and a unique rational number $0<\beta_{i}^{j} \leq 1$ such taht $\gamma_{i}^{j}=\mu_{i}^{j}+\beta_{i}^{j}$, for each $i=1, \ldots, r$. Define

$$
\xi_{j}=\sum_{i=1}^{r} \beta_{i}^{j} a_{i}=\sum_{i=1}^{r}\left(-\mu_{i}^{j}\right) a_{i}+\sum_{i=1}^{r} \gamma_{i}^{j} a_{i}=\sum_{i=1}^{r}\left(-\mu_{i}^{j}\right) a_{i}+\omega_{j} .
$$

Clearly $\xi_{j} \in \mathbf{G}(S) \cap$ intcon (S). It is straightforward to see that

$$
-\mu_{i}^{j}=\left\lfloor-\gamma_{i}^{j}\right\rfloor+1
$$

For example, let $S \subseteq \mathbb{N}^{3}$ and $\omega_{2}=\frac{3}{2} a_{1}+\frac{5}{7} a_{2}+\frac{13}{4} a_{3} \in \operatorname{Ap}(\mathrm{S}, \mathrm{E})$. Since $\gamma_{1}^{2}=$ $\frac{3}{2}, \gamma_{2}^{2}=\frac{5}{7}, \gamma_{3}^{2}=\frac{13}{4}$, we have $\mu_{1}^{2}=\left\lfloor-\frac{3}{2}\right\rfloor+1, \mu_{2}^{2}=\left\lfloor-\frac{5}{7}\right\rfloor+1, \mu_{3}^{2}=\left\lfloor-\frac{13}{4}\right\rfloor+1$, and so $\xi_{2}=-a_{1}-3 a_{3}+\omega_{2}$.

Lemma 1. Let $S$ be a simplicial affine semigroup. Then $f^{*} \in \mathrm{F}(\mathrm{S})$ if and only if $f^{*}+\xi_{k} \in S$, for every $k=1, \ldots, t$.

Proof. Let $f^{*}$ be a Frobenius vector for $S$. Since $\xi_{k} \in \mathbf{G}(S) \cap$ intcone $(S), f^{*}+\xi_{k} \in$ $S$. Conversely let $x=\sum_{i=1}^{r} z_{i} a_{i}+\omega_{l} \in \mathbf{G}(S) \cap \operatorname{intcone}(S)$. Since $x \in$ intcone $(S)$,

$$
z_{i}+\gamma_{i}^{l}>0 \Rightarrow z_{i}>-\gamma_{i}^{l} \Rightarrow z_{i} \geq\left\lfloor-\gamma_{i}^{l}\right\rfloor+1=-\mu_{i}^{l}
$$

Thus $x-\xi_{l}=\sum_{i=1}^{r}\left(z_{i}-\left(-\mu_{i}^{l}\right)\right) a_{i} \in S$ and so $f^{*}+x=\underbrace{f^{*}+\xi_{l}}_{\in S}+\underbrace{x-\xi_{l}}_{\in S} \in S$.

Theorem 2.1. Let $S$ be a simplicial affine semigroup. Then $\operatorname{MF}(S) \neq \varnothing$.

Proof. Let $f=\sum_{i=1}^{r} z_{i} a_{i}+\omega_{j} \in \mathbf{G}(S) \backslash S$. First of all, we observe that there exist $N_{1}, \ldots, N_{t} \in \mathbb{N}$ large enough such that

$$
\begin{aligned}
f+\sum_{k=1}^{t} N_{k} \xi_{k} & =\sum_{i=1}^{r} z_{i} a_{i}+\omega_{j}+\sum_{k=1}^{t} N_{k} \sum_{i=1}^{r} \beta_{i}^{k} a_{i}=\sum_{i=1}^{r} z_{i} a_{i}+\omega_{j}+\sum_{i=1}^{r} \sum_{k=1}^{t} N_{k} \beta_{i}^{k} a_{i} \\
& =\sum_{i=1}^{r}\left(z_{i}+\sum_{k=1}^{t} N_{k} \beta_{i}^{k}\right) a_{i}+\omega_{j} \in S .
\end{aligned}
$$

Now, if $f \notin \mathrm{F}(\mathrm{S})$, there exists $k_{1} \in\{1, \ldots, r\}$ such that $f_{1}=f+\xi_{k_{1}} \in \mathbf{G}(S) \backslash S$. If $f_{1} \notin \mathrm{F}(S)$, there exists $k_{2} \in\{1, \ldots, r\}$ such that $f_{1}=f+\xi_{k_{1}}+\xi_{k_{2}} \in \mathbf{G}(S) \backslash S$. Since this process can be repeated only finitely many times, by Lemma 1, we conclude that $\mathrm{F}(\mathrm{S}) \neq \varnothing$. Now we prove that $\operatorname{MF}(\mathrm{S}) \neq \varnothing$. Let $f=\sum_{i=1}^{r} z_{i} a_{i}+\omega_{j} \in \mathrm{F}(\mathrm{S}), f^{\prime}=\sum_{i=1}^{r} z_{i}^{\prime} a_{i}+\omega_{j^{\prime}} \in \mathrm{F}(\mathrm{S})$ and $f \in f^{\prime}+\operatorname{cone}(S)$. Then $z_{i}^{\prime}+\gamma_{i}^{j^{\prime}} \leq z_{i}+\gamma_{i}^{j}$ for all $i=1, \ldots, r$, and so $z_{i}^{\prime} \leq z_{i}+\gamma_{i}^{j}-\gamma_{i}^{j^{\prime}}$. On the other hand, since $\sum_{i=1}^{r} a_{i} \in \mathbf{G}(S) \cap \operatorname{intcone}(S)$, we have $f^{\prime}+\sum_{i=1}^{r} a_{i}=\sum_{i=1}^{r}\left(z_{i}^{\prime}+\gamma_{i}^{j^{\prime}}+1\right) \in S$, and thus $-\gamma_{i}^{j^{\prime}}-1 \leq z_{i}^{\prime}$. Hence $-\gamma_{i}^{j^{\prime}}-1<z_{i}^{\prime} \leq z_{i}+\gamma_{i}^{j}-\gamma_{i}^{j^{\prime}}$. The finiteness of $\operatorname{Ap}(\mathrm{S}, \mathrm{E})$ implies that $\left\{f^{*} \in \mathrm{F}(\mathrm{S}) \mid f \in f^{*}+\operatorname{cone}(\mathrm{S})\right\}$ is a finite set, which proves that $\operatorname{MF}(S) \neq \varnothing$. 
Definition 3. The simplicial affine semigroup $S$ is called pure simplicial if for each $i=1, \ldots, m, a_{r+i} \in$ intcone $(S)$. We abbreviate pure simplicial as $P$-simplicial.

We can define the following relation on $\mathbf{G}(S)$ : for any $a, b \in \mathbf{G}(S), a \leq_{S} b \Leftrightarrow$ $b-a \in S$. Let $\max (\operatorname{Ap}(S, E))=\left\{\eta_{1}, \eta_{2}, \ldots, \eta_{s}\right\}$ be the set of maximal elements of $\operatorname{Ap}(\mathrm{S}, \mathrm{E})$ with respect to $\leq_{S}$ and let $\gamma_{i}^{\max }=\left\lfloor\max _{j}\left(\gamma_{i}^{j}\right)\right\rfloor+1$, where $\max _{j}\left(\gamma_{i}^{j}\right)=$ $\max \left\{\gamma_{i}^{1}, \gamma_{i}^{2}, \ldots, \gamma_{i}^{t}\right\}$.

Theorem 2.2. Let $S$ be a simplicial affine semigroup.

(1) If $S$ is Cohen-Macaulay, then

$$
\mathrm{MF}(\mathrm{S}) \subset\left\{\sum_{i=1}^{r} z_{i} a_{i}+\omega \mid-1 \leq z_{i} \leq \gamma_{i}^{\max }, \omega \in \operatorname{Ap}(S, E)\right\} .
$$

(2) If $S$ is Cohen-Macaulay and P-simplicial, then

$$
\operatorname{MF}(S) \subset\left\{\sum_{i=1}^{r} z_{i} a_{i}+\eta \mid-1 \leq z_{i} \leq \gamma_{i}^{\max }, \eta \in \max (\operatorname{Ap}(S, E))\right\} .
$$

Proof. (1) Let $f=\sum_{i=1}^{r} z_{i} a_{i}+\omega \in \mathrm{F}(\mathrm{S})$. As $\sum_{i=1}^{r} a_{i} \in \mathbf{G}(S) \cap$ intcone(S), so $f+\sum_{i=1}^{r} a_{i}=\sum_{i=1}^{r}\left(z_{i}+1\right)+\omega \in S$. Hence by Proposition $2, z_{i} \geq-1$. Now let $z_{l}>\gamma_{l}^{\max }$ for some $l \in\{1, \ldots, r\}$. We show that $f \notin \operatorname{MF}(S)$. Set $f_{1}=\sum_{i=1, i \neq l}^{r} z_{i} a_{i}+\gamma_{l}^{\max } a_{l}+\omega$. Since $f \in f_{1}+\operatorname{cone}(S)$, it suffices to prove that $f_{1} \in \mathrm{F}(\mathrm{S})$. Let $x=\sum_{i=1}^{r} z_{i}^{\prime} a_{i}+\omega^{\prime} \in \mathbf{G}(S) \cap \operatorname{intcone}(S)$.

$$
\begin{aligned}
f_{1}+x & =\sum_{i=1, i \neq l}^{r} z_{i} a_{i}+\gamma_{l}^{\max } a_{l}+\omega+\sum_{i=1, i \neq l}^{r} z_{i}^{\prime} a_{i}+z_{l}^{\prime} a_{l}+\omega^{\prime} \\
& =\sum_{i=1, i \neq l}^{r}\left(z_{i}+z_{i}^{\prime}\right) a_{i}+\left(\gamma_{l}^{\max }+z_{l}^{\prime}\right) a_{l}+\left(\omega+\omega^{\prime}\right) .
\end{aligned}
$$

Since $f \in \mathrm{F}(\mathrm{S})$, we have $f+x=\sum_{i=1}^{r}\left(z_{i}+z_{i}^{\prime}\right) a_{i}+\omega+\omega^{\prime} \in S$. So by Proposition 2, $\sum_{i=1, i \neq l}^{r}\left(z_{i}+z_{i}^{\prime}\right) a_{i}+\omega+\omega^{\prime} \in S$. Clearly $\gamma_{l}^{\max }+z_{l}^{\prime}>0$, so $f_{1}+x$ is also in $S$ and therefore $f_{1} \in \mathrm{F}(\mathrm{S})$.

(2) Let $f=\sum_{i=1}^{r} z_{i} a_{i}+\omega \in \mathrm{F}(\mathrm{S})$. If $\omega \notin \max (\operatorname{Ap}(S, E))$, then there exists $\eta \in \max (\operatorname{Ap}(S, E))$ such that $\eta-\omega \in S$. Clearly $\eta-\omega \in \operatorname{Ap}(S, E)$, which implies that it belongs to intcone $(S)$, because $S$ is $P$-simplicial. Since $f \notin S$, by Proposition $2, f+\eta-\omega=\sum_{i=1}^{r} z_{i} a_{i}+\eta \notin S$. This contradicts $f \in \mathrm{F}(\mathrm{S})$.

By the previous theorem and Proposition 2, if $f^{*}=\sum_{i=1}^{r} z_{i} a_{i}+\eta$ is a minimal Frobenius vector of the Cohen-Macaulay $P$-simplicial semigroup $S$, then there exists $k \in\{1, \ldots, r\}$ such that $z_{k}=-1$ and therefore $f_{1}^{*}=-a_{k}+\sum_{i=1, i \neq k}^{r} \gamma_{i}^{\max } a_{i}+\eta$ is a Frobenius vector for $S$, because $f_{1}^{*} \in f^{*}+\operatorname{cone}(S)$.

Remark 1 (Numerical case). Every numerical semigroup $S=<a_{1}, \ldots, a_{m+1}>$ is a $P$-simplicial and Cohen-Macaulay semigroup. As a consequence of the above theorem $f^{*}(S)=-a_{1}+\max \left(\operatorname{Ap}\left(S, a_{1}\right)\right)$ (see [10, Proposition 2.12]).

As a consequence of the Theorem 2.2 and Lemma 1, we can compute the elements of $\mathrm{MF}(\mathrm{S})$ for Cohen-Macaulay simplicial semigroup, because we only have to check if a finite number of elements of $\mathrm{G}(S) \backslash S$ belongs to $\mathrm{MF}(\mathrm{S})$. 
Theorem 2.3. Let $S$ be a simplicial affine semigroup and $|\max (\operatorname{Ap}(S, E))|=1$. Then $f^{*}=\eta-\sum_{i=1}^{r} a_{i}$ is a Frobenius vector for $S$, where $\eta=\max (\operatorname{Ap}(S, E))$.

Proof. Let $x \in \mathbf{G}(S) \cap \operatorname{intcone}(S)$ and $f^{*}+x=\sum_{i=1}^{r} z_{i} a_{i}+\omega \in \mathbf{G}(S)$. So $\eta-\omega+x=\sum_{i=1}^{r}\left(z_{i}+1\right) a_{i}$. Since $\eta-\omega \in \operatorname{cone}(S)$ and $x \in \operatorname{intcone}(S)$, so $\eta-\omega+x \in \mathbf{G}(S) \cap$ intcone $(S)$. Hence for all $i=1, \ldots, r, z_{i}+1>0$, and so $z_{i} \geq 0$, consequently, $f^{*}+x \in S$.

Proposition 3. Let $S$ be a simplicial affine semigroup. The following statements are equivalent.

- $S$ is a Gorenstein semigroup;

- $S$ is a Cohen-Macaulay semigroup and the set $\operatorname{Ap}(S, E)$ has a unique maximal element.

Proof. Combining Theorem 4.6 with Theorem 2.8 in [11].

Corollary 1. Let $S$ be a Gorenstein simplicial affine semigroup. Then $f^{*}=\eta-\sum_{i=1}^{r} a_{i}$ is a Frobenius vector for $S$ where $\eta=\max (\operatorname{Ap}(S, E))$. Moreover, if $S$ is P-simplicial, then it is a minimal Frobenius vector for $S$ and it is unique.

In [1] (resp. [2]) it is shown that when $S$ is a complete intersection simplicial semigroup (resp. free semigroup) the vector $f^{*}=\eta-\sum_{i=1}^{r} a_{i}$ is the only minimal Frobenius vector for $S$. We recall that a simplicial affine semigroup is said to be free if $|\operatorname{Ap}(S, E)|=n_{1} n_{2} \cdots n_{m}$, where $n_{i}=\min \left\{\mathrm{k} \in \mathbb{N} \backslash 0 \mid \mathrm{ka}_{\mathrm{r}+\mathrm{i}} \in\left\langle\mathrm{a}_{1}, \mathrm{a}_{2}, \ldots\right.\right.$, $\left.\left.\mathrm{a}_{\mathrm{r}+\mathrm{i}-1}\right\rangle\right\}, \mathrm{i}=1, \ldots \mathrm{m}$. Clearly every simplicial affine semigroup with $m=1$ is free. Free semigoups are complete intersection and so they are Gorenstein. If $S$ be a free semigoup, then $I_{S}$ is generated by the set

$$
\left\{x_{r+1}^{n_{1}}-\prod_{i=1}^{r} x_{i}^{t_{1 i}}, x_{r+2}^{n_{2}}-\prod_{i=1}^{r+1} x_{i}^{t_{2 i}}, \ldots, x_{r+m}^{n_{m}}-\prod_{i=1}^{r+m-1} x_{i}^{t_{m i}}\right\}
$$

where $n_{i} a_{r+i}=\sum_{k=1}^{r+i-1} t_{i k} a_{k}$ (for more details, please see [12]).

Let $S$ be a Cohen-Macaulay $P$-simplicial semigroup. By Theorem 2.1, there exists at least one minimal Frobenius vector for $S$. Using the following algorithm we can compute minimal Frobenius vectors of $S$.

Algoritm : Computing minimal Frobenius vectors of a P-simplicial Cohen-Macaulay semigroup.

Inpute: A P-simplicial Cohen-Macaulay semigroup $S=\left\langle a_{1}, \ldots, a_{r+m}\right\rangle \subset \mathbb{N}^{r}$.

Output: The set of minimal Frobenius vectors of $S$.

Steps of the Algorithm:

1. Compute $I_{S}=\operatorname{ker} \varphi$ for $\varphi: K\left[x_{1}, \ldots, x_{r+m}\right] \rightarrow K[S], x_{i} \mapsto y^{a_{i}}$ ([6, Theorem 12.24]), ([13, Chapter 12]).

2. Compute a monomial K-basis $\left\{M_{i} \mid i\right\}$ of $\frac{K\left[x_{1}, \ldots, x_{r+m}\right]}{\left\langle I_{S}, x_{1}, \ldots, x_{r}\right\rangle}$ and set

$$
\operatorname{Ap}(S, E)=\left\{\omega_{1}, \omega_{2}, \ldots, \omega_{t}\right\}=\left\{\operatorname{deg}_{S}\left(M_{i}\right) \mid i\right\}
$$

3. Using (2.1) and (2.2), compute $\xi_{j}=\sum_{i=1}^{r}\left(-\mu_{i}^{j}\right) a_{i}+\omega_{j}, j=1, \ldots, t$.

4. Choose $\eta \in \max (\operatorname{Ap}(S, E))$ and set $A_{\eta}=\left\{-a_{1}-a_{2}-\cdots-a_{r}+\eta\right\}, \mathrm{MF}_{\eta}(\mathrm{S})=\varnothing$. 
5. Using Lemma 1, compute $T=\left\{t \in A_{\eta} \mid t \in \mathrm{F}(\mathrm{S})\right\}$. Set $\mathrm{MF}_{\eta}(\mathrm{S})=\mathrm{MF}_{\eta}(\mathrm{S}) \cup T$. Note that if $f+\xi_{k} \in S, k \in\{1, \ldots, t\}$, and $f \leq_{S} f^{\prime}$, then $f^{\prime}+\xi_{k} \in S$.

6. Set $A_{\eta}=\cup_{i=1}^{r}\left(a_{i}+\left(A_{\eta} \backslash T\right)\right) \backslash S$. We see that every element of $A_{\eta}$ is of the form $c_{1} a_{1}+\cdots+c_{r} a_{r}+\eta$.

7. Set $A_{\eta}=A_{\eta} \backslash\left\{c_{1} a_{1}+\cdots+c_{r} a_{r}+\eta \in A_{\eta} \mid c_{i}>\gamma_{i}^{\max }\right.$, for some $\left.i\right\}$ and repeat step 5.

8. The set of minimal Frobenius vectors of $S$ is equal to $\min _{\leq} \cup_{\eta} \mathrm{MF}_{\eta}(\mathrm{S})$.

Example 1. Let $S=\left\langle a_{1}=(1,5), a_{2}=(5,1), a_{3}=(2,2), a_{4}=(3,3)\right\rangle$. By Theorem $2.1, S$ has at least one minimal Frobenius vector. Performing the steps of the above algorithm we compute the set of minimal Frobenius vectors of $S$.

Step 1. Using $\operatorname{CoCo} A[3], K[S] \simeq \frac{K[x, y, z, w]}{I_{S}}$, where $I_{S}=\left\langle z^{3}-w^{2},-x y+w^{2}\right\rangle$.

Step 2. The set $\left\{1, \bar{z}, \bar{z}^{2}, \bar{w}, \bar{z} \bar{w}, \bar{z}^{2} \bar{w}\right\}$ is a monomial $K$-basis of $\frac{K[x, y, z, w]}{\left\langle I_{S}, x, y\right\rangle}$. Hence

$$
\operatorname{Ap}(S, E)=\left\{\omega_{1}=0, \omega_{2}=a_{3}, \omega_{3}=2 a_{3}, \omega_{4}=a_{4}, \omega_{5}=a_{3}+a_{4}, \omega_{6}=2 a_{3}+a_{4}\right\} .
$$

By Proposition 1, the semigroup $S$ is Cohen-Macaulay, because for every $x, y \in \operatorname{Ap}(S, E), x-y$ or $y-x$ is in intcone $(S)$ and since $a_{1}$ and $a_{2}$ are linearly independent, $x-y \notin \mathbf{G}\left(a_{1}, a_{2}\right)$.

Step 3. Using $\operatorname{CoCo} A$, we see that

$$
\begin{aligned}
& \omega_{1}=0 a_{1}+0 a_{2} \Rightarrow \xi_{1}=a_{1}+a_{2}=(6,6), \omega_{4}=\frac{1}{2} a_{1}+\frac{1}{2} a_{2} \Rightarrow \xi_{4}=a_{4}=(3,3) \\
& \omega_{2}=\frac{1}{3} a_{1}+\frac{1}{3} a_{2} \Rightarrow \xi_{2}=a_{3}=(2,2), \omega_{5}=\frac{5}{6} a_{1}+\frac{5}{6} a_{2} \Rightarrow \xi_{5}=a_{3}+a_{4}=(5,5) \\
& \omega_{3}=\frac{2}{3} a_{1}+\frac{2}{3} a_{2} \Rightarrow \xi_{3}=2 a_{3}=(4,4), \\
& \omega_{6}=\frac{7}{6} a_{1}+\frac{7}{6} a_{2} \Rightarrow \xi_{6}=-a_{1}-a_{2}+2 a_{3}+a_{4}=(1,1) .
\end{aligned}
$$

Step 4. Set $A_{2 a_{3}+a_{4}}=\left\{f^{*}=-a_{1}-a_{2}+2 a_{3}+a_{4}=(1,1)\right\}$.

Step 5. Since $f^{*}+\xi_{1}=2 a_{3}+a_{4} \in S, f^{*}+\xi_{2}=a_{4} \in S, f^{*}+\xi_{3}=a_{3}+a_{4} \in S$, $f^{*}+\xi_{4}=2 a_{3} \in S, f^{*}+\xi_{5}=2 a_{4} \in S$ and $f^{*}+\xi_{6}=a_{3} \in S$, so $f^{*} \in \mathrm{F}(\mathrm{S})$. Set $T=\left\{f^{*}\right\}$.

Step 6. $A_{2 a_{3}+a_{4}}=\cup_{i=1}^{2}(a_{i}+\underbrace{A_{2 a_{3}+a_{4} \backslash T}}_{\varnothing})=\varnothing$.

It follows that $f^{*}=(1,1)$ is the only minimal Frobenius vector for $S$. The semigroup $S$ is free, because $|\operatorname{Ap}(S, E)|=6$ (see Fig 1 ).

Example 2. Let $S=\left\langle a_{1}=(2,1), a_{2}=(1,5), a_{3}=(1,1), a_{4}=(4,5)\right\rangle$.

Step 1. Using $\operatorname{CoCo} A, K[S] \simeq \frac{K[x, y, z, w]}{I_{S}}$, where $I_{S}=\left\langle-z^{6}+x w, x^{3} y-z^{3} w\right.$, $\left.x^{2} y z^{3}-w^{2}\right\rangle$.

Step 2. The set $\left\{1, \bar{z}, \bar{z}^{2}, \bar{z}^{3}, \bar{z}^{4}, \bar{z}^{5}, \bar{w}, \bar{z} \bar{w}, \bar{z}^{2} \bar{w}\right\}$ is a monomial $K$-basis of $\frac{K[x, y, z, w]}{\left\langle I_{S}, x, y\right\rangle}$. Hence

$$
\begin{array}{r}
\operatorname{Ap}(S, E)=\left\{\omega_{1}=0, \omega_{2}=a_{3}, \omega_{3}=2 a_{3}, \omega_{4}=3 a_{3}, \omega_{5}=4 a_{3}\right. \\
\left.\omega_{6}=5 a_{3}, \omega_{7}=a_{4}, \omega_{8}=a_{3}+a_{4}, \omega_{9}=2 a_{3}+a_{4}\right\} .
\end{array}
$$

Since $a_{1}$ and $a_{2}$ are linearly independent, one obtains that if $x, y \in \operatorname{Ap}(S, E)$ and $x \neq y$, then $x-y \notin \mathbf{G}\left(a_{1}, a_{2}\right)$. Hence by Proposition $1, S$ is a Cohen-Macaulay 


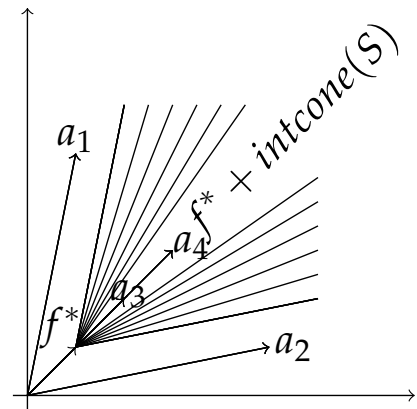

Fig.1

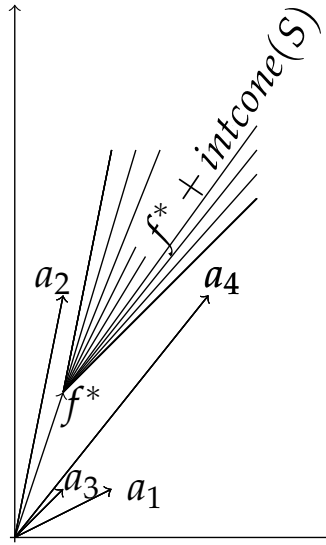

Fig.2

semigroup but not Gorenstein, because $\max (\operatorname{Ap}(S, E))=\left\{\eta_{1}=2 a_{3}+a_{4}, \eta_{2}=5 a_{3}\right\}$ (see Proposition 3 ).

Step 3. Using CoCoA, we see that

$$
\begin{aligned}
& \omega_{1}=0 a_{1}+0 a_{2} \Rightarrow \xi_{1}=a_{1}+a_{2}=(3,6), \omega_{2}=\frac{4}{9} a_{1}+\frac{1}{9} a_{2} \Rightarrow \xi_{2}=a_{3}=(1,1) \\
& \omega_{3}=\frac{8}{9} a_{1}+\frac{2}{9} a_{2} \Rightarrow \xi_{3}=2 a_{3}=(2,2), \omega_{4}=\frac{12}{9} a_{1}+\frac{3}{9} a_{2} \Rightarrow \xi_{4}=-a_{1}+3 a_{3}=(1,2) \\
& \omega_{5}=\frac{16}{9} a_{1}+\frac{4}{9} a_{2} \Rightarrow \xi_{5}=-a_{1}+4 a_{3}=(2,3), \\
& \omega_{6}=\frac{20}{9} a_{1}+\frac{5}{9} a_{2} \Rightarrow \xi_{6}=-2 a_{1}+5 a_{3}=(1,3) \\
& \omega_{7}=\frac{5}{3} a_{1}+\frac{2}{3} a_{2} \Rightarrow \xi_{7}=-a_{1}+a_{4}=(2,4), \\
& \omega_{8}=\frac{19}{9} a_{1}+\frac{7}{9} a_{2} \Rightarrow \xi_{8}=-2 a_{1}+a_{3}+a_{4}=(1,4) \\
& \omega_{9}=\frac{23}{9} a_{1}+\frac{8}{9} a_{2} \Rightarrow \xi_{9}=-2 a_{1}+2 a_{3}+a_{4}=(2,5) .
\end{aligned}
$$

Step 4. Set $A_{2 a_{3}+a_{4}}=\left\{f_{1}=-a_{1}-a_{2}+2 a_{3}+a_{4}=(3,1)\right\}$.

Step 5. We have $f_{1}+\xi_{1}=2 a_{3}+a_{4} \in S, f_{1}+\xi_{2}=2 a_{1} \in S, f_{1}+\xi_{3}=2 a_{1}+a_{3} \in S$, $f_{1}+\xi_{4}=a_{1}+2 a_{3} \in S, f_{1}+\xi_{5}=a_{1}+3 a_{3} \in S$,

$f_{1}+\tilde{\xi}_{6}=4 a_{3} \in S, f_{1}+\xi_{7}=5 a_{3} \in S, f_{1}+\xi_{8}=a_{4} \in S$ and $f_{1}+\xi_{9}=2 a_{1}+a_{2} \in S$. So $f_{1} \in \mathrm{F}(\mathrm{S})$ and like in the example above $\mathrm{MF}_{2 \mathrm{a}_{3}+\mathrm{a}_{4}}=\left\{f_{1}\right\}$.

Now we use the algorithm for $\eta_{2}=5 a_{3}$. Set $A_{5 a_{3}}=\left\{f_{2}=-a_{1}-a_{2}+5 a_{3}=\right.$ $(2,-1)\}$. As $f_{2}+\xi_{2}=(3,0) \notin S, f_{2} \notin \mathrm{F}(S)$. Step 6 yields $A_{5 a_{3}}=\left\{f_{3}=-a_{1}+\right.$ $\left.\eta_{2}=(3,4), f_{4}=-a_{2}+\eta_{2}=(4,0)\right\}$. Since $f_{3}+\xi_{4}=(4,6) \notin S$ and $f_{4}+\xi_{4}=$ $(5,2) \notin S, f_{3}$ and $f_{4}$ are not in $\mathrm{F}(\mathrm{S})$. Using the algorithm, $A_{5 a_{3}}=\left\{f_{5}=-a_{1}+a_{2}+\right.$ $\left.\eta_{2}=(4,9), f_{6}=a_{1}-a_{2}+\eta_{2}=(6,1)\right\}$. As $\gamma_{1}^{\max }=3$ and $\gamma_{2}^{\max }=1$, we go back to Step 5. The vectors $f_{5}$ and $f_{6}$ are not in $\mathrm{F}(S)$ because $f_{5}+\xi_{4}=(5,11) \notin S$ and $f_{6}+\xi_{4}=(7,3) \notin S$. Using steps 6 and $7, A_{5 a_{3}}=\left\{f_{7}=2 a_{1}-a_{2}+\eta_{2}=(8,2)\right\}$. As $f_{7}+\xi_{4}=(9,4) \notin S$, so $f_{7} \notin \mathrm{F}(\mathrm{S})$. Set $A_{5 a_{3}}=\left\{f_{8}=3 a_{1}-a_{2}+\eta_{2}=(10,3)\right\}$. Since $f_{8}+\xi_{4}=(11,5) \notin S$, so $f_{8} \notin \mathrm{F}(\mathrm{S})$. Finally, $A_{5 a_{3}}=\varnothing$ ( steps 6 and 7). Hence $f_{1}$ is the only minimal Frobenius vector for $S$ (see Fig 2).

\section{Acknowledgement}

The authors are very grateful to the anonymous referee for carefully reading the paper and for his or her comments and suggestions which have improved the paper. 


\section{References}

[1] A. Assi, P. A. Garcia-Sanchez and I. Ojeda, Frobenius vectors, Hilbert series and gluing, Journal of Commutative Algebra, 7 (2015), no. 3, 317-335.

[2] A. Assi, The Frobenius vector of a free affine semigroup. J. Algebra Appl. 11 (2012), no. 4, 1250065.

[3] CoCoATeam, CoCoA: A system for doing Computations in Commutative Algebra (2011). Available at http:/ / cocoa.dima.unige.it

[4] R. Fröberg, The Frobenius number of some semigroups, Comm. Algebra 22 (1994), 6021-6024.

[5] J. Herzog, Generators and relations of abelian semigroups and semigroup rings. Manuscripta Math., 3 (1970). 175-193.

[6] E. Miller and B. Sturmfels, Combinatorial commutative algebra, Vol. 227, Springer-Verlag, New York, 2005.

[7] J. L. Ramirez Alfonsín, The diophantine Frobenius problem, Equipe Combinatoire Universite Pierre et Marie Curie, 2005.

[8] O. J. Rödseth, On a linear Diophantine problem of Frobenius, J. reine angew. Math. 301 (1978), 171-178.

[9] J. C. Rosales, P. A. García-Sánchez, Finitely generated commutative monoids. Nova Science Publishers, Inc., Commack, NY, 1999.

[10] J. C. Rosales, P. A. García-Sánchez, Numerical Semigroups, Developments in Mathematics, 20. Springer, New York, 2009.

[11] J. C. Rosales, P. A. García-Sánchez, On Cohen-Macaulay and gorenstein simplicial affine semigroups, Proc. Edinburgh Math. Soc. (2) 41 (1998) 517-537.

[12] J. C. Rosales, P. A. García-Sánchez, On free affine semigroups, Semigroup Forum 58 (1999) 367-385. Archiv der Mathematik 83 (2004), 488-496.

[13] B. Sturmfels, Gröbner bases and convex polytopes, Univ. Lecture Ser., Vol. 8, Amer. Math. Soc., Providence, RI, 1995.

[14] J.J. Sylvester, Problem 7382, Educational Times 37 (1884), 26; reprinted in: Mathematical questions with their solution, Educational Times 41(1884), 21.

[15] B. Vizvári, Generation of uniformly distributed random vectors of good quality, Rutcor Research Report (1994), No. RRR 1793. 
[16] B. Vizvári, On a generalization of the Frobenius problem, Technical Report MN/30 Computer and Automation Inst., Hungarian Academy of Sciences (1987).

Department of Pure Mathematics,

Faculty of Mathematics and Computer Science,

Amirkabir University of Technology, Tehran, Iran

E-mail addresses: a_mahdavi@aut.ac.ir ,frahmati@aut.ac.ir 